\title{
Potential role of endothelial cell surface ectopic redox complexes in COVID-19 disease pathogenesis
}

\author{
Author: Isabella Panfoli ${ }^{\mathrm{A}}$
}

The novel coronavirus infectious disease (COVID-19) has rapidly spread and poses a great challenge to researchers, both in elucidating its pathogenic mechanism and developing effective treatments. It has been recently proposed that COVID-19 is an endothelial disease. Indeed, the COVID-19 virus binds to angiotensin-converting enzyme type 2 (ACE2), which is expressed in endothelial cells. ACE2 could be implicated in the production of reactive oxygen species (ROS) caused by endothelial dysfunction due to viral damage. Consequently, oxidative stress could prime these cells to acquire a pro-thrombotic and pro-inflammatory phenotype, predisposing patients to thromboembolic and vasculitic events and to disseminated intravascular coagulopathy (DIC). This implies a pivotal role played by oxygen in the pathogenetic mechanism of COVID-19 disease, in that its availability would tune the oxidant state and consequent damage.

KEYWORDS: COVID-19, ectopic electron transfer chain, endothelium, oxidative stress, oxygen

DOI: $10.7861 /$ clinmed.2020-0252

The role of the endothelium in COVID-19

Severe acute respiratory syndrome coronavirus 2 (SARS-CoV-2) emerged at the end of 2019 as a new type of highly pathogenic coronavirus of still unclear biological aetiology that rapidly spread around the world. Now COVID-19 illness is recognised as a global epidemic,' bearing an alarming mortality index and necessitating extensive control measures. However, due to the novelty of this disease in humans, and the lack of definitive clinical, instrumental and autoptic data, the pathogenic mechanisms of COVID-19 disease have not been fully elucidated.

It is clear that COVID-19 is not simply an interstitial pneumonia, because evidence points to its being a systemic thrombotic and thrombo-embolic syndrome affecting both the venous and arterial circulations. ${ }^{2}$ Many patients display clinical manifestations of shock and symptoms of microcirculation dysfunction. Death often occurs due to thromboembolism, multiple organ failure (MOF) and sepsis associated with disseminated intravascular coagulation (DIC). ${ }^{2}$

The resting endothelium produces antithrombotic molecules, including nitric oxide (NO) and prostacyclin ( $\mathrm{PGI}_{2}$ ). Endothelial

Author: Aassociate professor, University of Genoa, Genoa, Italy dysfunction (ED) in COVID-19 ${ }^{3}$ is primarily caused by oxidative stress, ${ }^{4}$ a systemic condition associated with decreased NO and expression of prothrombotic and cell-surface adhesion molecules, and which plays a pivotal role in both sepsis and DIC. ${ }^{5}$ There is a tight relationship between $\mathrm{ED}$, inflammation, reactive oxygen species (ROS) production and increased risk of thromboembolism. ROS stimulate expression of tissue factor (TF) and can also directly inactivate protein $\mathrm{C}$, a major anticoagulant.

Accordingly, it has recently been proposed that COVID-19 primarily targets the endothelium. ${ }^{6}$ Clinical and preclinical evidence in COVID-19 patients support the hypothesis that the endothelium is a key target organ in the disease, leading to the consequent multi-organ systemic manifestations. ${ }^{6}$ The hypothesis of COVID-19 as an endothelial disease appears interestingly unifying for its systemic manifestations, as it would account for the involvement of the nervous system, heart, liver and kidney ${ }^{1}$ in COVID-19, the lung just being the most sensitive target. The virus primary receptor is angiotensin-converting enzyme 2 (ACE2), which is expressed in the airway cells, intestinal epithelial cells, kidney cells, cerebral neurons and alveolar monocytes, but also in the endothelial cells (ECs) that line the vascular lumen; these are the most widespread cells in the body and play a pivotal role in vascular homeostasis. ${ }^{5}$ SARS-CoV-2 binding with ACE2 could cause over-inflammatory and pro-thrombotic disseminated events. COVID-19 binds to human ACE2 in the general population, with patients with diabetes and cardiovascular disease being at high risk. ${ }^{6}$ Concern has arisen regarding whether angiotensin receptor blockers (ARBs) and ACE inhibitors (ACEIs) increase the morbidity and mortality of COVID-19, as ARBs and ACEI upregulate ACE2 expression.? There are conflicting data on this topic: it has recently been suggested that the ARB could play a protective effect against COVID-19 pneumonia. Notably, a confounding parameter is old age, a strong predictor of COVID-19-related death, as elderly people are often treated with ARBs or ACEIs. To date there is no conclusive evidence for ARBs or ACEIs increasing the risk or being beneficial for COVID-19; therefore it is not recommended to discontinue these medications.?

\section{The role of oxidative stress in COVID-19}

Oxidative stress and an impaired antioxidant system have been reported to be linked to the pathogenesis and severity of coronavirus infections. ${ }^{8}$ Redox balance impairment may trigger inflammatory responses. In SARS-CoV-1 infection, the viral 3C-like protease $\left(3 \mathrm{CL}^{\mathrm{pro}}\right)$ was demonstrated to increase ROS production in

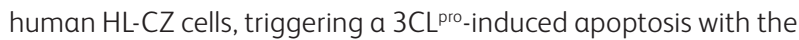
involvement of NF- $\mathrm{KB}$ signalling. ${ }^{9}$ 


\section{Possible source of oxidative stress}

ROS are mainly generated as by-products of cellular aerobic metabolism. The mitochondrial electron transfer chain (ETC) complexes I and III are the major source of ROS..$^{10}$ Intracellularly, a minor source of ROS production is the activity of cytosolic enzymes, including neutrophil NADPH oxidases. However, the ultimate source of a massive extracellular ROS production is less clear. The ETC and $F_{1} F_{0}$-ATP synthase (ATP synthase) are expressed outside the mitochondrion, as suggested by the frequency with which these proteins are found in ectopic locations by proteomics. ${ }^{11}$ An extra-mitochondrial oxidative phosphorylation has been reported to occur in rod outer segment disks,,$^{12}$ myelin,,$^{13}$ exosomes, ${ }^{14}$ plasma membranes of cancer cells, ${ }^{15}$ human umbilical vein endothelial cells (HUVECs) ${ }^{16}$ and hepatocytes. ${ }^{17}$ A cell surface ATP synthase was also found in lipid rafts of ECs plasma membranes, where it was active in ATP synthesis. ${ }^{18}$

Thus, ROS production inside and possibly outside the endothelial cell, enhanced by hyperglycaemia, could cause the systemic damage seen in COVID-19. ED is a key determinant in cardiovascular disease and diabetes mellitus, which are frequent comorbidities in COVID-19 patients. Insulin resistance could cause abnormalities in virus pathogenesis, increasing mortality risk. ${ }^{7}$ Moreover, SARS-CoV-2 may exert a direct action on the pancreatic beta cells expressing ACE2. Metformin has been shown to improve endothelial function. ${ }^{19}$ A high percentage of COVID-19 deaths came from patients with altered glucose homeostasis, in turn related to higher expression of IL-6. Such higher plasma IL-6 levels in hyperglycemic patients would be responsible for the reduced effects of tocilizumab, which targets IL-6 receptors, in these patients..$^{20}$ Optimal glycemic control is a good strategy to ameliorate outcomes in high-risk patents ${ }^{7}$ and it would be advisable to assess the impact of hypoglycaemic therapies on COVID-19 patients.?

The answer to the question of why viral damage causes microvascular inflammation and thrombosis in predisposed individuals would be that a source of ROS (and oxidative stress) in the vessel lumen is the ETC in the plasma membrane of the ECs. The COVID-19 pathogenic mechanism would be a vascular luminal oxidative stress produced by the ectopic ETC in the plasma membrane of the ECs, impaired by viral damage. This would in turn prime microvascular thrombosis. A pivotal role for oxygen as a trigger of ROS production by the impaired ETC can be therefore envisaged. This would explain the primary involvement of those organs that are more exposed to oxygen: the lung but also the kidney and the nervous system. If oxygen availability to the impaired ectopic ETC expressed in the plasma membrane of the ECs plays a primary role in COVID-19 pathogenesis, mechanical ventilation might require a finer tuning of oxygen fluxes. The use of costly resources such as prolonged invasive ventilation would also benefit, in economic terms, from a deeper knowledge of the pathogenesis of the most severe disease complications.

\section{References}

1 Rothan HA, Byrareddy SN. The epidemiology and pathogenesis of coronavirus disease (COVID-19) outbreak. J Autoimmun 2020;109:102433.

2 Ciceri F, Beretta L, Scandroglio AM et al. Microvascular COVID-19 lung vessels obstructive thromboinflammatory syndrome
(MicroCLOTS): an atypical acute respiratory distress syndrome working hypothesis. Crit Care Resusc 2020, in press.

3 Klok F, Kruip M, van der Meer et al. Incidence of thrombotic complications in critically ill ICU patients with COVID-19. Thromb Res 2020;191:145-7.

4 Pham-Huy LA, He H, Pham-Huy C. Free radicals, antioxidants in disease and health. Int J Biomed Sci 2008;4:89-96.

5 Incalza MA, D'Oria R, Natalicchio A et al. Oxidative stress and reactive oxygen species in endothelial dysfunction associated with cardiovascular and metabolic diseases. Vascul Pharmacol 2018;100:1-19.

6 Sardu C, Gambardella J, Morelli MB et al. Hypertension, thrombosis, kidney failure, and diabetes: is COVID-19 an endothelial disease? A comprehensive evaluation of clinical and basic evidence. J Clin Med 2020;9:1417.

7 Sardu C, D'Onofrio N, Balestrieri ML et al. Outcomes in patients with hyperglycemia affected by Covid-19: can we do more on glycemic control? Diabetes Care 2020, in press (doi: 10.2337/dc200723).

8 Delgado-Roche L, Mesta F. Oxidative stress as key player in severe acute respiratory syndrome coronavirus (SARS-CoV) infection. Arch Med Res 2020;51:384-7.

9 Lin C-W, Lin K-H, Hsieh T-H, Shiu S-Y, Li J-Y. Severe acute respiratory syndrome coronavirus 3C-like protease-induced apoptosis. FEMS Immunol Med Microbiol 2006:46:375-80.

10 Genova ML, Pich MM, Bernacchia A et al. The mitochondrial production of reactive oxygen species in relation to aging and pathology. In: Mitochondrial pathogenesis. Springer, 2004:86-100.

11 Panfoli I, Ravera S, Bruschi M, Candiano G, Morelli A. Proteomics unravels the exportability of mitochondrial respiratory chains. Expert Rev Proteomics 2011;8:231-9.

12 Bruschi M, Petretto A, Caicci F et al. Proteome of bovine mitochondria and rod outer segment disks: commonalities and differences. J Proteome Res 2018;17:918-25.

13 Ravera S, Bartolucci M, Garbati P et al. Evaluation of the acquisition of the aerobic metabolic capacity by myelin, during its development. Mol Neurobiol 2016;53:7048-56.

14 Panfoli I, Ravera S, Podestà M et al. Exosomes from human mesenchymal stem cells conduct aerobic metabolism in term and preterm newborn infants. FASEB J 2016;30:1416-24.

15 Chang HY, Huang HC, Huang TC et al. Ectopic ATP synthase blockade suppresses lung adenocarcinoma growth by activating the unfolded protein response. Cancer Res 2012;72:4696-706.

16 Arakaki N, Nagao T, Niki R et al. Possible role of cell surface H+ -ATP synthase in the extracellular ATP synthesis and proliferation of human umbilical vein endothelial cells. Mol Cancer Res 2003;1:931-9.

17 Mangiullo R, Gnoni A, Leone A. Structural and functional characterization of $F(0) F(1)-A T P$ synthase on the extracellular surface of rat hepatocytes. Biochim Biophys Acta 2008;1777:1326-35.

18 Yamamoto K, Shimizu N, Obi S et al. Involvement of cell surface ATP synthase in flow-induced ATP release by vascular endothelial cells. Am J Physiol Heart Circ Physiol 2007;293:H1646-53.

19 Hamidi Shishavan M, Henning RH, Van Buiten A et al. Metformin improves endothelial function and reduces blood pressure in diabetic spontaneously hypertensive rats independent from glycemia control: comparison to vildagliptin. Sci Rep 2017;7:1-12.

20 Marfella R, Paolisso P, Sardu C et al. Negative impact of hyperglycemia on Tocilizumab therapy in COVID-19 patients. medRxiv 2020;2020.04.29.20076570.

Address for correspondence: Prof Isabella Panfoli, University of Genoa, School of Medical and Pharmaceutical SciencesDIFAR, Viale Benedetto XV, 3, 16132 Genoa, Italy. Email: panfoli@difar.unige.it 